\title{
Evolution without standing genetic variation: change in transgenerational plastic response under persistent predation pressure
}

\author{
Arnaud Sentis $\mathbb{D}^{1,2} \cdot$ Raphaël Bertram ${ }^{1} \cdot$ Nathalie Dardenne $^{1} \cdot$ Felipe Ramon-Portugal $^{1} \cdot$ Gilles Espinasse $^{1}$. \\ Ines Louit ${ }^{1}$ - Lucie Negri ${ }^{1}$ - Elena Haeler ${ }^{3,4}$ - Thomas Ashkar ${ }^{1}$ - Théo Pannetier ${ }^{1}$ - James L. Cunningham ${ }^{1}$. \\ Christoph Grunau $^{5} \cdot$ Gaël Le Trionnaire ${ }^{6} \cdot$ Jean$^{-C h r i s t o p h e}$ Simon $^{6} \cdot$ Alexandra Magro $^{1} \cdot$ Benoit Pujol $^{1}$. \\ Jean-Louis Hemptinne ${ }^{1} \cdot$ Etienne Danchin ${ }^{1}$
}

Received: 15 November 2017 / Revised: 22 May 2018 / Accepted: 3 June 2018 / Published online: 29 June 2018

(c) The Genetics Society 2018

\begin{abstract}
Transgenerational phenotypic plasticity is a fast non-genetic response to environmental modifications that can buffer the effects of environmental stresses on populations. However, little is known about the evolution of plasticity in the absence of standing genetic variation although several non-genetic inheritance mechanisms have now been identified. Here we monitored the pea aphid transgenerational phenotypic response to ladybird predators (production of winged offspring) during 27 generations of experimental evolution in the absence of initial genetic variation (clonal multiplication starting from a single individual). We found that the frequency of winged aphids first increased rapidly in response to predators and then remained stable over 25 generations, implying a stable phenotypic reconstruction at each generation. We also found that the high frequency of winged aphids persisted for one generation after removing predators. Winged aphid frequency then entered a refractory phase during which it dropped below the level of control lines for at least two generations before returning to it. Interestingly, the persistence of the winged phenotype decreased and the refractory phase lasted longer with the increasing number of generations of exposure to predators. Finally, we found that aphids continuously exposed to predators for 22 generations evolved a significantly weaker plastic response than aphids never exposed to predators, which, in turn, increased their fitness in presence of predators. Our findings therefore showcased an example of experimental evolution of plasticity in the absence of initial genetic variation and highlight the importance of integrating several components of non-genetic inheritance to detect evolutionary responses to environmental changes.
\end{abstract}

These authors contributed equally: Jean-Louis Hemptinne and Etienne Danchin.

Electronic supplementary material The online version of this article (https://doi.org/10.1038/s41437-018-0108-8) contains supplementary material, which is available to authorized users.

Arnaud Sentis

Arnaud.sentis@sete.cnrs.fr

1 Laboratoire Évolution and Diversité Biologique (EDB UMR 5174), Université Fédérale de Toulouse Midi-Pyrénées, CNRS, IRD, UPS, Toulouse, France

2 Ecological Networks and Global Change Group, Experimental and Theoretical Ecology Station, UMR5321, CNRS, University Paul Sabatier, Moulis, France

3 Forest Science; Bern University of Applied Sciences - School of Agricultural, Forest and Food Sciences HAFL, Zollikofen 3052, Switzerland

\section{Introduction}

Identifying genetic and non-genetic mechanisms of parentoffspring resemblance (i.e., inclusive heritability) is central to evolutionary biology because without them, selection

4 Landscape Ecology; Institute of Terrestrial Ecosystems; ETH Zürich, Zürich 8092, Switzerland

5 University of Perpignan Via Domitia, IHPE UMR 5244, CNRS, IFREMER, University of Montpellier, Perpignan F-66860, France

6 UMR 1349; IGEPP (Institut de Génétique, Environnement et Protection des Plantes); INRA, Agrocampus Ouest, Université Rennes 1; Domaine de la Motte B.P. 35327, Le Rheu cedex F35653, France 
cannot lead to evolution (Danchin et al. 2011; Day and Bonduriansky 2011). Yet most studies focus solely on genetic variants. Evidence for non-genetic mechanisms of transmission between parents and offspring (i.e., heredity) is accruing at a fast pace. Nongenetic mechanisms of inheritance include cultural, ecological and epigenetic inheritance, as well as parental effects and niche construction, all of which potentially contributing to heredity (Bossdorf et al. 2008; Danchin et al. 2011; Bonduriansky 2012; Danchin 2013; Huneman and Walsh 2017; Wang et al. 2017). For instance, experimentally induced DNA methylation changes can explain $60-90 \%$ of heritability estimates in the flowering time of some Arabidopsis thaliana isogenic lines (Cortijo et al. 2014). The existence of non-genetic inheritance mechanisms raises the question of their importance for adaptive evolution.

Phenotypic plasticity, the capacity of a single genotype to produce different phenotypes in different environments (Whitman and Agrawal 2009), plays a crucial role in the ability of populations to adapt to rapidly changing environments (Chevin et al. 2010; Donelson et al. 2011; Munday et al. 2013; Sentis et al. 2015). Phenotypic plastic responses to environmental changes are common (Agrawal 2001; West-Eberhard 2003; Whitman and Agrawal 2009; Huey et al. 2012). They usually occur through the modulation of physiology, morphology, reproduction, and behaviour allowing organisms to cope with abrupt changes (Donelson et al. 2011; Gardner et al. 2011; Huey et al. 2012), and have important consequences for community and ecosystem functioning (Miner et al. 2005; Sentis et al. 2015; Sentis et al. 2017a). Interestingly, there is mounting evidence that environmentally induced phenotypic changes can sometimes be transmitted across generations (Danchin et al. 2011; Donelson et al. 2011; Rechavi et al. 2011; Schott et al. 2014; Dias et al. 2015; Klosin et al. 2017). This body of evidence is accounting for a significant non-genetic component of inclusive heritability (i.e., heritable phenotypic changes not directly due to variation in the DNA sequence (Danchin and Wagner 2010)). For instance, when mice were conditioned to fear a usually benign odour, at least their offspring and grand offspring were born fearing it in the absence of any conditioning (Dias and Ressler 2014). This example is one among many others showing that the transmission of the environmentally induced phenotype can be driven by epigenetic mechanisms involving chromatin structure modifications, mediated by histone posttranslational modifications, DNA methylation, and small noncoding RNAs (siRNAs and piRNAs), which in turn affect gene expression (Turner 2009; Feil and Fraga 2012; Klosin et al. 2017). Non-genetically inherited, environmentally induced phenotypes can increase the resistance/ response to biotic and abiotic stresses, probably improving offspring fitness (Whittle et al. 2009; Boyko et al. 2010).
Moreover, these induced phenotypic changes can sometime persist long after the environmental stress ceased. For instance, temperature-induced expression of an artificial transgenic construct can persist for at least 14 generations in Caenorhabditis elegans (Klosin et al. 2017). Environmentally induced phenotypes that persist and are inherited across generations thus offer a potential target for natural selection (Turner 2009). However, the role of phenotypic plasticity and the inheritance of environmentally induced phenotypes in adaptive evolution, their importance relative to genetic variants, and their stability when environmental induction has ceased remain relatively unknown (but see Klosin et al. 2017 and Danchin et al. 2018).

Plastic phenotypic changes in response to a singleenvironmental modification that subsequently remains constant can lead to the stable maintenance of the environmentally induced phenotypic variants through at least two different mechanisms with very different potential evolutionary consequences. First, the reconstruction of the plastic response at every generation can yield to a constant frequency of phenotypic (non-genetic) variants upon which selection can act. Consistent plastic responses across generations have the potential to modify the speed of genetically based adaptive evolution. In particular, when plasticity is non-adaptive, it places populations further away from their fitness optimum but strengthens directional selection acting on genetic variation (Ghalambor et al. 2007; Ghalambor et al. 2015). The second mechanism maintaining environmentally induced phenotypic variants across generations is the inheritance of the phenotype that was environmentally induced (Danchin et al. 2011; Klosin et al. 2017). In either case, selection might act upon this source of transgenerational variation (Pujol et al. 2018). The stable maintenance of environmentally induced phenotypic variants can potentially lead to the loss of plasticity by canalization (Lande 2009; Herman et al. 2014; Kronholm and Collins 2016). Additionally, genetically based adaptive evolution might be enhanced by environmentally induced epigenetic changes that are maintained across generations and increase the rate of genetic mutation because this is potentially leading to genetic assimilation (Herman and Sultan 2011; Herman et al. 2014; Kronholm and Collins 2016; Pocheville and Danchin 2017). Overall, both the reconstruction of the plastic response and the inheritance of the environmentally induced phenotype can lead to a stable frequency of non-genetic variants and have contrasting consequences on evolutionary changes by selection. Moreover, little is known about the stability of these mechanisms and their long-term effects over multiple generations.

When environmental conditions vary across generations but the conditions met by parents are good predictors of the offspring's environment, transgenerational phenotypic 
plasticity (i.e., parent individuals alter specific developmental traits in their progeny in response to particular environmental stresses (Herman and Sultan 2011; Holeski et al. 2012; Donelson et al. 2018)) is expected to be positively selected if it improves the fitness of offspring (Herman et al. 2014). An increasing number of studies hypothesised that transgenerational plasticity is important for species adaptation to rapid environmental change (Holeski et al. 2012; Luquet and Tariel 2016; Sentis et al. 2017b). However, as most studies investigated transgenerational plastic responses over a small number of generations (Burton and Metcalfe 2014; Donelson et al. 2018), their role in the adaptive evolution of populations over larger timescales remains rarely documented. In a twogenerations experiment, Luquet and Tariel (2016) showed that transgenerational plasticity can reduce withingeneration plasticity and lead to a switch from a plastic towards a constitutive expression of induced defenses in a freshwater snail. However, other short-term experiments showed that transgenerational plasticity does not always affect within-generation plasticity (Burton and Metcalfe 2014; Donelson et al. 2018). Collectively, these findings suggest that a modification of the transgenerational plastic response, and ultimately the constitutive expression of the induced phenotype, has the potential to emerge when the exposure of a population to environmental stressors over multiple generations maintains a high frequency of nongenetic phenotypic variants through long-term stable reconstruction. As this hypothesis remains untested we adopted an experimental evolution approach to test it in the pea aphid Acyrthosiphon pisum (Harris).

The pea aphid is emerging as an ideal biological system to study epigenetics and phenotypic plasticity (Srinivasan and Brisson 2012) and test the transgenerational hypotheses mentioned above. Under long-day conditions, it reproduces asexually by apomictic parthenogenesis (i.e., oocytes are parthenogenetically produced without meiosis or recombination). The pea aphid asexual phase generates a stochastic and/or environment-oriented repertoire of epigenetic variants with distinct behavioural and physiological traits (Dombrovsky et al. 2009; Pasquier et al. 2014). After acquisition, these epigenetic and phenotypic variants can be inherited across generations (de Vos et al. 2010; Walsh et al. 2010), and artificially selected (Andrade and Roitberg 1995; Pasquier et al. 2014). Transgenerational plastic responses to environmental factors are common in aphids and play an important role in their life-cycle and population dynamics (Srinivasan and Brisson 2012; Sentis et al. 2017b). A spectacular example of aphid transgenerational phenotypic plasticity is the asexual production of winged offspring in response to predators or crowding (Dixon and Agarwala 1999; Srinivasan and Brisson 2012). Interestingly, this ability to produce winged offspring depends on the phenotype of the mother as winged mothers tend to produce less winged offspring than wingless mothers (Sutherland 1970; MacKay 1977). Moreover, this refractory phase can persist up to two aphid generations (Sutherland 1970), which suggests that exposure to stress can influence winged aphid frequencies for up to three generations with a high-winged frequency at the first generation followed by low-winged frequencies for the next two generations. Previous studies on molecular mechanisms reported that this extreme and discrete transgenerational phenotypic response (i.e., the production of winged offspring) is under the control of the ecdysone hormone and might involve alternative DNA methylation patterns at specific genome locations (Zera 2003; Brisson 2010; Pasquier et al. 2014; Vellichirammal et al. 2016; Vellichirammal et al. 2017). Transgenerational plasticity in wing production thus provides an opportunity to study the evolution of a trait based on epigenetically inherited information.

Here we experimentally investigated non-genetic evolution in the transgenerational phenotypic response of pea aphids to ladybird predators that consists in the asexual production of winged offspring in response to predators. We chose an experimental evolution approach as it is suited for detecting phenotypic changes across generations and deciphering non-genetically based changes (Johannes et al. 2008; Kawecki et al. 2012). One of the main originality of our approach is that we erased standing genetic variation from our initial experimental population by initiating all experimental populations from a single parthenogenetic female. By diminishing initial genetic variation in this way, we increased our chances to isolate non-genetic effects from potential genetic effects (to the exception of new DNA mutations that are expected to be rare in our setting). We monitored population density and the proportion (hereafter, frequency) of winged individuals in aphid experimental populations (hereafter, lines) with or without ladybird predators for 27 generations. We aimed to elucidate whether (1) the frequency of the winged phenotype would change across generations in response to permanent predation, (2) the transgenerational plastic response to predators would evolve in the constant presence of predators over the course of multiple generations, and (3) the environmentally induced phenotype would persist after predator removal, and whether such persistence would change over the course of generations exposed to predators.

Theory predicts that inducible defences evolve only if benefits outweigh costs (Tollrian and Harvell 1999; Agrawal 2001). In other words, for wing forms to be selected for, the benefits of producing wings in the presence of predators should outweigh the costs of wings. In a preliminary experiment, we found that the survival of winged and unwinged adult aphids did not significantly differ $(p=0.75)$ according to whether they were in the presence or absence of 
predators over a $24 \mathrm{~h}$ period in a setting similar to the one used in the main experiment (i.e., in closed experimental cages preventing long distance dispersal). However, the development of wings is known to be costly because winged aphids have a longer developmental time and fecundity is lower in winged than unwinged individuals (Dixon et al. 1993). As a consequence, the costs of developing wings are expected to outweigh the benefits when dispersal is unsuccessful as it is the case in our spatially unstructured experimental system. We thus expected winged offspring production to be selected against in our enclosed experimental system preventing dispersal (see below for more details). The frequency of winged aphids should therefore decrease in the experimental lines submitted to a recurrent predation stress, which might ultimately lead to the complete loss of inducible defences (i.e., the loss of plasticity), with the wingless phenotype eventually becoming constitutive.

\section{Material and methods}

\section{Experimental system}

The experimental system was a three-level food chain: the predatory ladybird Harmonia axyridis (Pallas), the pea aphid A. pisum, and the broad bean Vicia faba (L). Approximately 200 adults of $H$. axyridis were collected in October 2015 at Auzeville-Tolosane (433'N, $1^{\circ} 29^{\prime} \mathrm{E}$, South of France), brought to the laboratory, reared in $5000-\mathrm{cm}^{3}$ plastic boxes, and fed ad libitum three times a week with pea aphids and pollen. Corrugated filter paper was added to each box to provide a suitable substrate for oviposition. $H$. axyridis eggs were collected three times a week and neonate larvae were reared in $175-\mathrm{cm}^{3}$ plastic boxes and fed pea aphids ad libitum before experiments. A stock colony of the pea aphid LSR1 clonal lineage was maintained at our laboratory at low density on broad bean grown from seeds (Ets Henrion s.a.; Belgium, cv. Aquadulce) in nylon cages $(30 \times 30 \times 30 \mathrm{~cm})$. Using a clonal lineage allowed us to estimate phenotypic changes of fixed genotype, and thereby phenotypic plasticity. The LSR1 clonal lineage was chosen because (1) it only harbours the obligate endosymbiont Buchnera aphidicola, thus avoiding the potential confounding effect of temporal variation in symbiont community composition, (2) a preliminary experiment revealed that it responds to predation by $H$. axyridis larvae by asexually producing more winged offspring than in the absence of predation, and (3) under our laboratory rearing conditions it produces one generation every 7 days which allows conducting a multigenerational experiment in a relatively short time (for a multicellular organism). All insects and plants were maintained in climate chambers $\left(\right.$ Dagard $\left.^{\circledR}\right)$ at $21 \pm 1{ }^{\circ} \mathrm{C}, 50-60 \%$ relative humidity, and under a $16 \mathrm{~L}: 8 \mathrm{D}$ photoperiod to mimic spring conditions during which the pea aphid only reproduces by apomictic parthenogenesis, offspring thus being clones of their mother.

\section{Experimental design}

A single LSR1 founder individual started the evolution experiment (Fig. S1). After four generations of clonal amplification 9 lines of $\sim 5000$ aphids each were obtained from the single founder (i.e., no initial standing genetic variation). Each of these nine lines was then divided between two experimental evolution treatments (Fig. S1). The first experimental evolution treatment was the exposure of aphids to predators at every generation over the 27 generations of the experimental evolution (hereafter referred to as the Evolutionary Predation Treatment). The second treatment was the absence of predators to which control lines were submitted over the period of the whole experimental evolution (hereafter referred to as Evolutionary Control Treatment). As a result, we surveyed a total of 18 lines for 27 generations (Fig. S1). Throughout the experiment, aphid populations were maintained in cages $(37 \mathrm{~cm}$ high; $\varnothing 20.5 \mathrm{~cm}$ ) containing 20 eight-day-old $V$. faba plants in $5 \mathrm{~L}$ plastic pots filled with $4 \mathrm{~L}$ of fertilized soil substrate ( ${ }^{\circledR}$ Jiffy substrates NFU 44-551). The pots were enclosed in a plastic frame $(37 \mathrm{~cm}$ high; $\varnothing 20.5 \mathrm{~cm})$ covered with a tightmeshed net preventing aphids or ladybirds to escape. During the experiment, bean plants were watered twice a week with $750 \mathrm{~mL}$ of tap water per pot. For the evolutionary predation treatment, two second-instar larvae of $H$. axyridis were introduced in each of the nine cages $24 \mathrm{~h}$ after the introduction of the aphids.

After 7 days (broadly corresponding to one pea aphid generation under our experimental conditions), we removed the predators, harvested the plants, collected all aphids using a fine paintbrush. Once all aphids were collected from an experimental line, we then quickly transferred them to a petri dish $(50 \times 9 \mathrm{~mm})$ and weighed their total biomass with a micro-balance $\left(10^{-7} \mathrm{~g}, \mathrm{SC} 2\right.$, Sartorius $\left.{ }^{\circledR}\right)$. We then collected two random aphid samples per line. The first one of $600 \mathrm{mg}$ ( 750 aphids) was deep frozen at $-80{ }^{\circ} \mathrm{C}$ and later used to count the demographic structure (number of winged adults, wingless adults, and nymphs) under a stereoscopic microscope. The second random sample of $300 \mathrm{mg}(\sim 375$ aphids) was transferred into a new experimental cage containing 20 new plants standardized as mentioned above to initiate the next generation. A preliminary experiment showed that an aphid biomass of $300 \mathrm{mg}$ yielded, after 7 days, a population size of $\sim 3600$ aphids which is (1) large enough to minimize extinction risk due to stochasticity or predation pressure and (2) small enough to minimize resource competition linked to plant depletion. Twenty-four hours after transferring the $300 \mathrm{mg}$ aphid sample, two second-instar larvae of the ladybird predator were 
introduced in each of the nine new cages of the predation treatments. This procedure was repeated 27 times (i.e., for 27 generations).

To test the persistence of the changes in the winged aphid frequency of the evolutionary predation treatment, we initiated one branch line for each of the nine experimental lines of the evolutionary predation treatment (i.e., each predation line was duplicated using an additional $300 \mathrm{mg}$ sample to seed each branch line) at three different times during the experimental evolution (Fig. S1). The main line was used to keep the experimental evolution line running with predators, whereas the branch line was used to test the persistence of the plastic response after removing the predation pressure. For that goal, we immediately removed predators in the branch lines and maintained these lines for five generations with the same protocol as the main lines, but without predators (Fig. S1). These parallel predator removal experiments were initiated after generations 3,13 , and 22 (i.e., generations 4, 14, and 23 were the first generations without predators) to investigate the level of persistence of the observed changes at different stages of the experimental evolution.

\section{Additional experiments comparing the fitness and transgenerational plasticity of experimental evolution lines in presence and absence of predators}

Two independent additional experiments were conducted after 16 and 25 generations of experimental evolution to compare the fitness (i.e., population growth rate) and the transgenerational plasticity of "naive aphids" from Control lines not exposed to predators during the experimental evolution and "experienced aphids" from Predation lines continuously exposed to predators during the experimental evolution (Fig. S1). For both additional experiments, we used the same full factorial design including the two Evolutionary Treatments (Predation and Control without predators) from the experimental evolution $\times$ two Test Predation Treatments (i.e., presence/absence of predators during the additional experiments). To remove potential maternal and grand-maternal effects associated with predation exposure over the course of the experimental evolution, so that the likelihood to have aphids in their refractory phase would be extremely low, we used aphid populations from the control lines and the branch lines for which predators had been removed three generations earlier. In other words, we used aphid populations from the branch and control lines at generations 16 and 25 to start these additional experiments (Fig. S1). To confirm the feasibility of these experiments, we conducted analyses comparing the proportion of winged aphids at generation 16 and 25 in the control and branch lines. They revealed no significant generation effects (Generation: $F_{1,21.4}=0.6692, N=36, p$ $=0.42$ ). No significant statistical interaction with the control/branch treatment was found (Evolutionary Treatment $x$ Generation: $F_{1,21.4}=0.21, N=36, p=0.65$ ). These side results indicate that the composition of winged and unwinged aphids was similar when we started both additional experiments, thus minimizing the potential confounding maternal and grand-maternal effects. We used the same experimental method for these two experiments: from each of the 18 experimental evolution lines, we collected two random aphid samples of $75 \mathrm{mg}$ which were immediately transferred into transparent plastic cylinders $(\varnothing: 14 \mathrm{~cm}$; $h$ : $29 \mathrm{~cm}$ ) containing four new plants standardized as mentioned above. This process yields 36 experimental cylinders per experiment (i.e., two cylinders for each of the 18 experimental evolution lines). The sample size (i.e., $75 \mathrm{mg}$ ) was chosen to obtain a similar aphid density per plant as in the large cages used for the experimental evolution. One day after introducing the aphids, we introduced one first-instar larvae of ladybird $H$. axyridis in half of the 36 cylinders (i.e., in one of the two experimental cylinders of each experimental evolution line) resulting in 9 replicates for each combination of Evolutionary Predation Treatments and Test Predation Treatments. After 8 days, we removed the ladybird larvae, collected the aphids, and weighed their total biomass. We then collected a random aphid sample of $75 \mathrm{mg}$ which was then immediately transferred into a new transparent plastic cylinder containing four new plants. This second transfer allowed us to continue the experiment for a period long enough to test for the transgenerational plastic response to predators while keeping aphid density sufficiently low to avoid the depletion of the plant resource. One day later, one ladybird larva was introduced in half of the cylinders according to their assigned predation treatment. The other 18 cylinders were used as controls without predators. After 8 days, we removed the ladybird larvae, collected the aphids, weighed their total biomass and counted the number of winged adults, wingless adults, and nymphs under a stereoscopic microscope. During the experiments, temperature and humidity were recorded continuously using Hobo U12 $\left(\mathrm{Hobo}^{\circledR}\right)$ units.

\section{Statistical analyses}

We tested for the effects of the Evolutionary Predation Treatment, the Generation (as a continuous variable), and their interaction on the frequency (i.e. proportion) of winged aphids, and on aphid population biomass growth rate with LMMs. The growth rate of the populations biomass was calculated as $\log \left(B_{\mathrm{f}} / B_{\mathrm{i}}\right) / d$ where $B_{\mathrm{i}}$ and $B_{\mathrm{f}}$ are the initial (i.e. $300 \mathrm{mg}$ ) and final (i.e., at the time of transfer to a new generation) aphid biomasses and $d$ is the number of days between two measurements (i.e., 7). In these analyses, 
winged aphid frequencies were arcsine square root transformed to linearize their binomial distribution (Gotelli and Ellison 2004). Line nested within evolutionary predation treatments was included in the models as a random effect to account for repeated measurement across generations. The significance of the fixed model terms was assessed using Kenward-Roger $F$-tests. Post hoc Tukey tests were used to determine significant differences among means.

We next investigated the effects of the frequency of winged aphids, the Evolutionary Predation Treatment, and their interaction on aphid population biomass growth rate with a LMM (i.e., an ANCOVA including the line nested within evolutionary predation treatments as a random effect to account for repeated measures across generations). The significance of the fixed model terms was assessed using Kenward-Roger $F$-tests. Post hoc Tukey tests were used to determine significant differences among slopes and intercepts.

To investigate the persistence of transgenerational effects, we analysed the effects of Predator Removal Treatment (i.e., two treatments were included in the analyses: the control never exposed to predators [in black in Fig. S 1 and Fig. 1] and the treatment with predators removed [in blue in Fig. S1 and Fig. 1]), Generation (as a categorical variable) and their interaction on the arcsine square root frequency of winged aphids and on aphid population biomass growth rate with LMMs. Line nested within evolutionary predation treatments was included in the models as a random effect to account for repeated measures across generations. The significance of the fixed model terms was assessed using Kenward-Roger $F$-tests.
Post hoc Tukey tests were used to determine significant differences among means.

For the additional experiments in which we compared experimental evolution lines after 16 and 25 generations of experimental evolution, we analysed (separately for generation 16 and 25) the effects of the Evolutionary Predation Treatment (i.e., the presence/absence of predators during experimental evolution), the Test Predation Treatment (i.e., the presence/absence of predators during the additional experiment) and their interaction on the arcsine square root frequency of winged aphids and on aphid population biomass growth rate with LMMs. The identification number of each evolutionary line was included in the models as a random effect to account for the fact that some replicates originated from the same evolutionary line (Fig. S1). The significance of the fixed model terms was assessed using Kenward-Roger $F$-tests. Post hoc Tukey tests were used to determine significant differences among means. LMMs were computed using the lme4 package (Bates et al. 2015) in R 3.4.1 (R Development Core Team 2017).

\section{Results}

\section{Change in the frequency of winged aphids}

As expected, we found that the predator presence increased the frequency of winged aphids in the progeny relative to unexposed individuals. This resulted in very high frequencies of winged aphids at the second generation (Fig. 1). After this first phase of increase, the frequency of winged
Fig. 1 Proportions of winged adult aphids (mean \pm SE) across generations of the experimental evolution with predators (in red), without predators (in black) and in branch lines for which predators were removed after generations 3,13 , and 22 (in blue). "*”" or "NS" denote the significance $(P<0.05$, or $P>$ 0.05 , respectively) of differences between controls (without predator, black dots) and branch lines after predator removal (blue dots). The vertical black dotted line indicates the time of initial predator introduction in the treatment lines

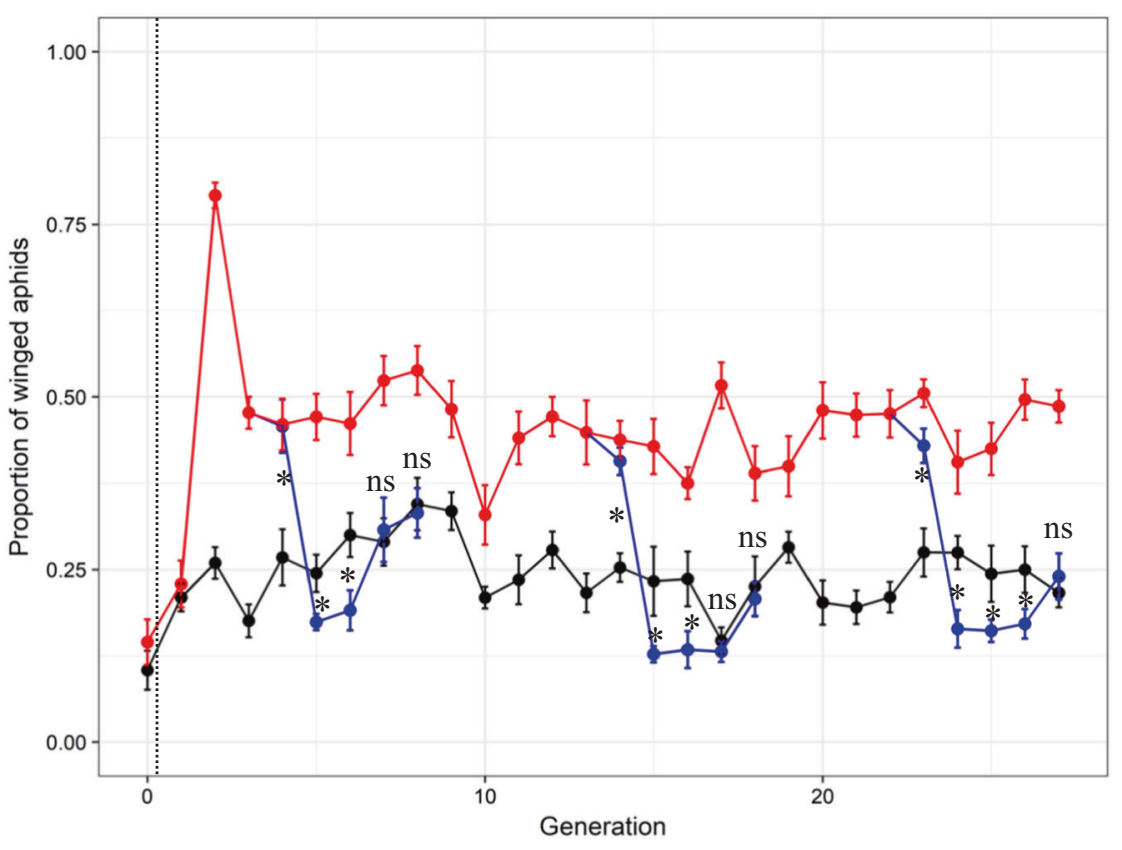


aphid decreased and then stabilised around 0.5 for the lines exposed to predators. Our analyses revealed that winged frequency was significantly higher in the presence of predators (Evolutionary Predation Treatment: $F_{1,388.76}=46.32$, $N=486, p<0.0001$; Fig. 1 and S2) and that this predation effect did not significantly change with the increasing number of generations (Evolutionary Predation Treatment $\times$ Generation: $F_{1}, 466=0.007, \quad N=486, \quad p=0.93$ ). Finally, the frequency of winged aphids remained stable across generations for both treatments as indicated by the lack of significant Generation effect $\left(F_{1,466}=2.34, N=\right.$ 486, $p=0.13$; Fig. 1 and S2).

In the branch lines where predators were removed (in blue in Fig. 1 and S2), frequencies of winged aphid remained approximately twice as high as the control without predators for the first generation after predator removal (i.e. generations 4, 14, and 23). Winged frequencies then dropped below the level of the control lines (in black in Fig. 1) for at least two generations before catching up with the control level (Fig. 1). When comparing the lines of the main experiment with persistent predation pressure (in red in Fig. 1) to the branch lines where predators were removed at three different stages of the experimental evolution (in blue in Fig. 1) we found that winged aphid frequencies decreased faster at the third, and thereby later, stage of predator removal than at to the first two stages (Fig. S3 and text S1). When comparing control lines without predators (in black in Fig. 1) with branch lines where predators were removed (in blue in Fig. 1), we found that the interaction between Predation Treatment and Generation was significant when analysing the relationship with the frequency of winged adult aphids $\left(F_{14,225.54}=5.13, N=270, p<0.0001\right)$. Post hoc tests indicated that winged aphid frequencies were significantly higher in the branch lines than in the control lines for the first generation after predator removal and were then significantly lower than the control for two generations in branches starting at generations 3 or 13 or for three generations in branches starting at generation 22 before matching the control level (Fig. 1, comparison of blue and black dots). In other words, our analyses indicated that winged aphid frequency came back to the control level at the fourth generation after predator removal for the first and second branch lines, whereas for the third branch lines, it took five generations for winged frequency to come back to control level. Overall, the number of generations needed for winged aphid frequency to come back to the control level after stopping predation increased over the course of the experimental evolution (Fig.1).

A more detailed analysis of these data revealed that the number of generations needed for the frequency of winged aphids to come back to the control level after predator removal tended to increase proportionally to the number of generations exposed to predators (Text S2 and Fig. 2, linear regression analysis: $y=3.83+0.037 \mathrm{x}, R^{2}=0.97$, $p=0.07)$.

\section{Population growth rate}

Predators significantly decreased aphid population growth rate $\left(F_{1,388.76}=75.73, N=486, p<0.0001\right.$; Fig. 3 and S4), and this effect was similar across generations as the interaction between Evolutionary Predation Treatment (i.e., the presence/absence of predators during experimental evolution) and Generation was not significant $\left(F_{1,466}=1.91, N\right.$ $=486, p=0.167)$. In fact the difference between treatments remained similar in a context where population growth rate significantly decreased across generations both in the presence and absence of predators $\left(F_{1,466}=133.22, N=486\right.$, $p<0.0001$, slope value $=-0.0026$ ).

When comparing control lines (in black in Fig. 3) to the lines where predators were removed (in blue in Fig. 3), we found that the population growth rate depended on the generation at which an independent branch line was founded (i.e., the generation at which predators were removed), as illustrated by the significant interaction between the Evolutionary Predation Treatment and the Generation $\left(F_{14,223.54}\right.$ $=6.98, N=270, p<0.0001$ ). After predator removal, the population growth rate in the branch line increased quickly and went back to the level of the control after three generations when predators were removed at the third generation, whereas it took only two generations to match control level for the two following predator removals (Fig. 3).

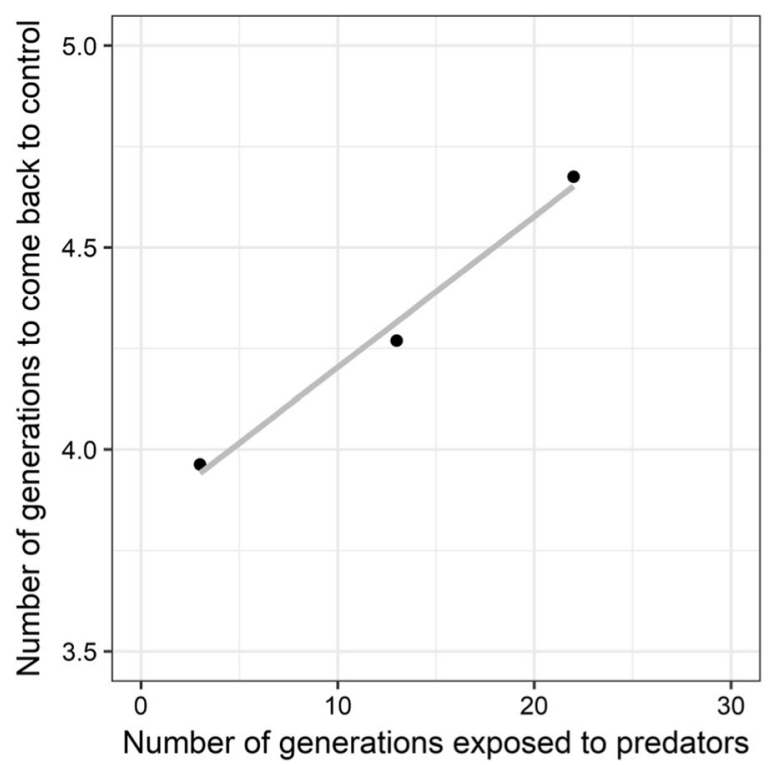

Fig. 2 Relationship between the number of generations exposed to predators and the number of generations needed for the proportion of winged aphids to come back to the control level after predator removal (see Text S2 for more details about the method used to estimate $y$-axis values for each predator removal) 


\section{Relationship between winged aphids and population growth rate}

Aphid population growth rate (Fig. 4) significantly varied with the frequency of winged aphids but this effect depended on the Evolutionary Predator Treatments (Winged Aphid Frequency $\times$ Evolutionary Predator Treatment: $F_{4}$, $604.51=7.24, N=621, p<0.0001$; Fig. 4). For the treatments of the main lines with predators (Fig. 4a) and the branch lines (Fig. 4b), we found a negative relationship between winged aphid frequency and the population growth rate: the higher the winged morph frequency, the lower the growth rate. This relationship was significant for all treatments except the main control line without predators (main line with predators: $F_{1,239.94}=13.38, N=243, p=0.0003$, main line without predator: $F_{1,239.15}=2.96, N=243, p=$ 0.0867 (black line in Fig. 4), first branch line: $F_{1,38.29}=$ 4.56, $N=45, p=0.0392$, second branch line: $F_{1,37.95}=$ 52.76, $N=45, p<0.0001$, third branch line: $F_{1,41.19}=$ $41.19, N=45, p<0.0001)$. Post hoc analyses indicated that the intercept of the relationship between winged aphid frequency and population growth rate was lower for lines continuously exposed to predators than for unexposed control lines $\left(F_{1,392.58}=34.45, N=486, p<0.0001\right.$; Fig. $4 a)$ indicating that the presence of predators reduced population growth rates.

Interestingly, we found that multigenerational exposure to predator gradually changed the relationship between winged aphid frequency and population growth rate (Fig. 4b). The slope and intercept of this relationship became respectively steeper and higher with the number of generations exposed to predators $\left(F_{2,125.72}=13.87, N=\right.$ 135, $p<0.0001$; Fig. 4b).

\section{Additional experiments comparing the fitness and transgenerational plasticity of experimental evolution lines in presence and absence of predators}

In the first additional experiment, the lines that were separated at generation 16 from the experimental evolution during which they were exposed or unexposed to predators (Evolutionary Predation Treatment and Evolutionary Control Treatment) were characterised by similar plastic responses to predators. We found that (1) winged aphids were more frequent in the presence of predators (Test Predation Treatment: $F_{1,14.26}=211.09, N=31, p<0.0001$; Fig. 5a) and (2) this effect did not depend on whether aphids were naive-not exposed to predators during the experimental evolution - or experienced - continuously exposed to predators during the experimental evolution-(Test Predation Treatment $\times$ Evolutionary Predation Treatment: $F_{1}$, $14.26=1.78, N=31, p=0.203$; Fig. 5a). Moreover, the Evolutionary Predation Treatment had no significant effect on wing frequency $\left(F_{1}, 14.80=0.119, N=31, p=0.735\right)$ indicating that wing frequency in this additional experiment was independent of the presence or absence of predators during experimental evolution. Similarly, we found that the effect of predators on aphid population growth rate during the additional experiment was independent from whether aphids were naive or experienced, as illustrated by the lack of significance of the statistical interaction between Evolutionary Predation Treatment and Test Predation Treatment
Fig. 3 Aphid population growth rate $($ mean $\pm \mathrm{SE})$ across generations in the experimental evolution with predators (in red), without predators (in black) and in the branch lines for which predators were removed after generations 3, 13, and 22 (blue lines). "*" and "NS" denote significance $(P<0.05$, or $P>$ 0.05 , respectively) of differences between the control without predator (black dots) and the treatment in which predators were removed (blue dots) at a given generation. The vertical black dashed line indicates the time of initial predator introduction in treatment lines

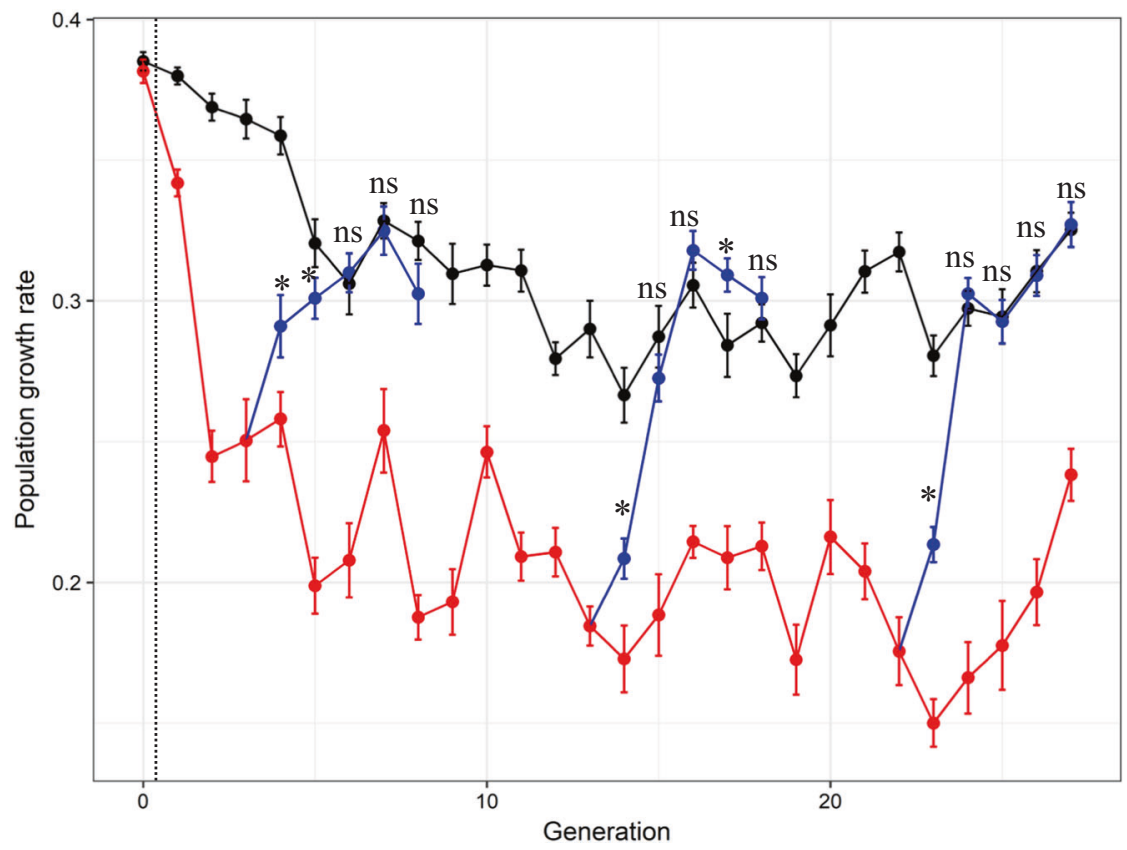


Fig. 4 Relationship between the proportion of winged adult aphids and the population growth rate in the main experimental lines (a) without (black dots and full black line) and with predators (red dots and dashed red line), and (b) in the branch experimental lines for which predators were removed after generations 3 (light blue dots and full light blue line), 13 (blue dots and short-dashed blue line), and 22 (dark blue dots and long-dashed dark blue line). Repeated measures correlation tests (Bakdash and Marusich 2017): main experimental lines without predators $(r=-0.11 \mathrm{df}$ $=233, p=0.09$ ); main experimental lines with predators $(r=-0.23, \mathrm{df}=233$, $p=0.0004)$; branch experimental lines for which predators were removed after generations $3(r=-0.38, \mathrm{df}=$ $35, p=0.02), 13(r=-0.79, \mathrm{df}$ $=35, p<0.0001)$, and $22(r=$ $-0.73, \mathrm{df}=35, p<0.0001)$

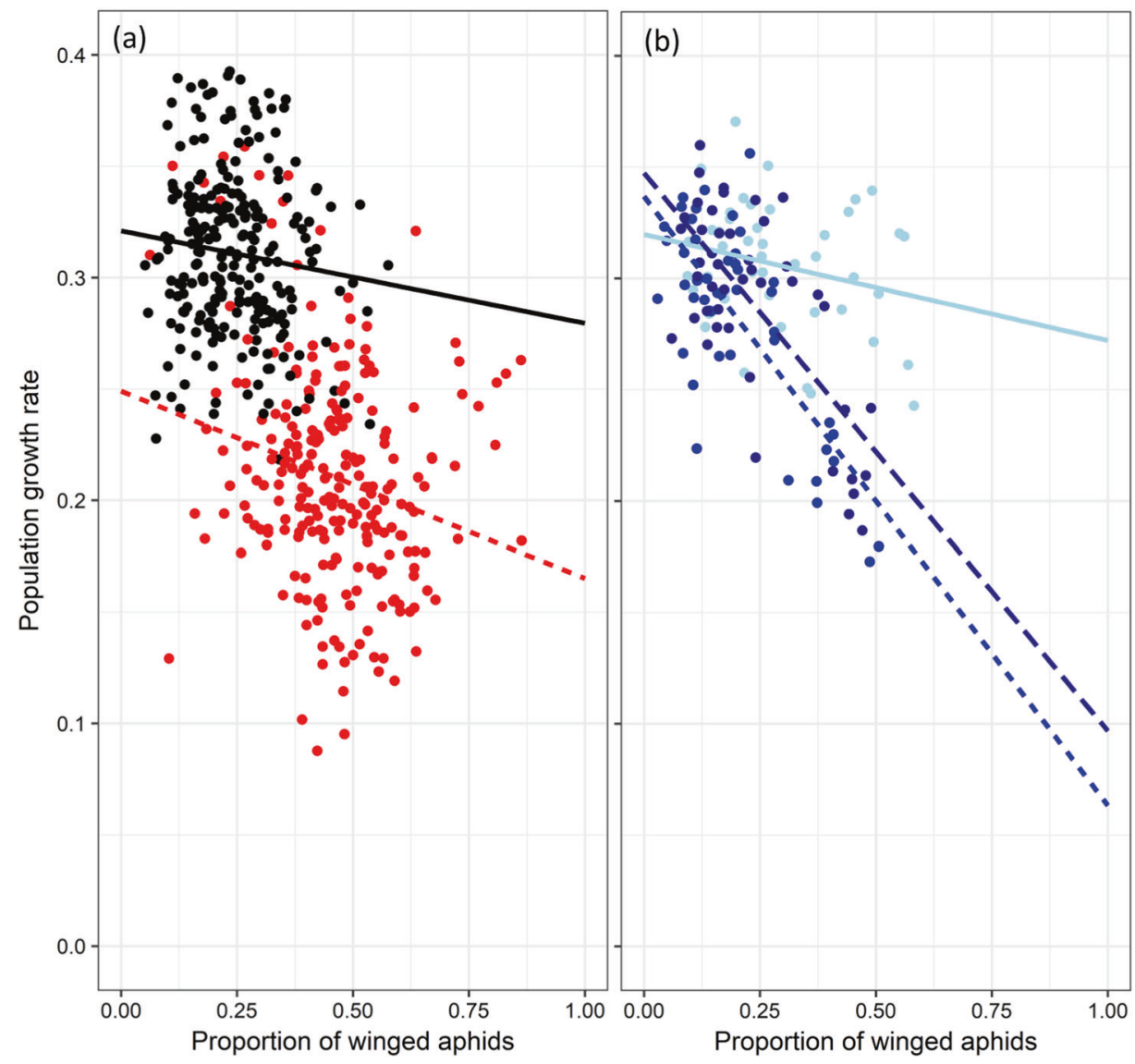

$\left(F_{1,13.63}=1.84, N=31, p=0.1974\right.$; Fig. 5c $)$. Moreover, population growth rate did not significantly differ between the two experimental evolution treatments (Evolutionary Predation Treatment: $F_{1,15.32}=0.083, N=31, p=0.7768$ ) but the presence of predators significantly decreased aphid population growth rate (Test Predation Treatment: $F_{1,13.63}$ $=83.385, N=31, p<0.0001)$. Overall, our results indicate that the continuous presence of predators during experimental evolution did not result in a change of the population growth rate or of the plastic response to predators at generation 13 of the evolution experiment.

Contrastingly, in the second additional experiment performed at generation 25 of the experimental evolution, naive aphids (not exposed to predators during the experimental evolution) and experienced aphids (continuously exposed to predators during the experimental evolution) showed different plastic responses to predators (Test Predation Treatment $\times$ Evolutionary Predation Treatment: $F_{1}$, $12.054=6.38, N=28, p=0.0265$; Fig. 5b). While the frequency in winged aphid significantly increased in presence of predators for both Evolutionary Treatments, the magnitude of this effect was weaker for experienced aphids compared to naive aphids (Fig. 5b). Similarly, at this late stage in the evolution experiment, we found that the effect of predators on aphid population growth rate during the additional experiment depended on whether aphids were naive or experienced, as illustrated by the significance of the statistical interaction between Evolutionary Predation Treatment and Test Predation Treatment $\left(F_{1,13.02}=12.24\right.$, $N=28, p=0.0038)$. The presence of predators during the additional experiment significantly decreased aphid population growth rate but the magnitude of this effect was weaker for experienced aphids compared to naïve aphids (Fig. 5d). Overall, our results indicate that the continuous presence of predators during experimental evolution decreased the aphid transgenerational phenotypic response to predators and increased their population growth rate at generation 25 of the evolution experiment. This contrasted with the results observed at generation 16 .

\section{Discussion}

Phenotypic plasticity constitutes a fast non-genetic response that can increase the ability of organisms to cope with rapidly changing environments (Chevin et al. 2010; Donelson et al. 2011; Munday et al. 2013; Sentis et al. 2015). Additionally, when the induced phenotype is nongenetically inherited across generations, this can provide populations with an alternative source of adaptive 
Fig. 5 Change in aphid plastic response and population growth rate between the 16th and the 25th generation of experimental evolution. Here we illustrate the differences in plasticity in the additional experiments for experimental lines that were isolated after 16 and 25 generations of experimental evolution (first and second column, respectively). Changes in proportions of winged adult aphids (mean $\pm \mathrm{SE}$ ) are represented in $\mathbf{a}$ and $\mathbf{b}$, and changes in aphid population growth rates (mean $\pm \mathrm{SE}$ ) are represented in $\mathbf{c}$ and $\mathbf{d}$. The plastic response is observed across environments (absence and presence of predators corresponding to the Test Predation Treatment represented on the $x$-axes) by comparing the change in the phenotype values represented along the $y$-axes for aphids continuously exposed or never exposed to predators (red and black dots, respectively) during experimental evolution. “*” or "NS" denote significance $(P<0.05$, or $P>0.05$, respectively) of differences between evolutionary predation treatments (i.e., the presence/ absence of predators during experimental evolution)

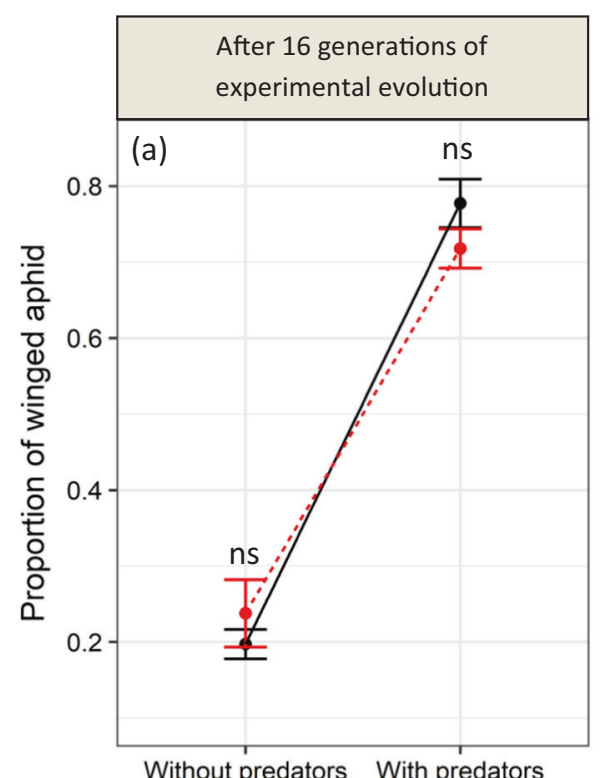

Without predators With predators

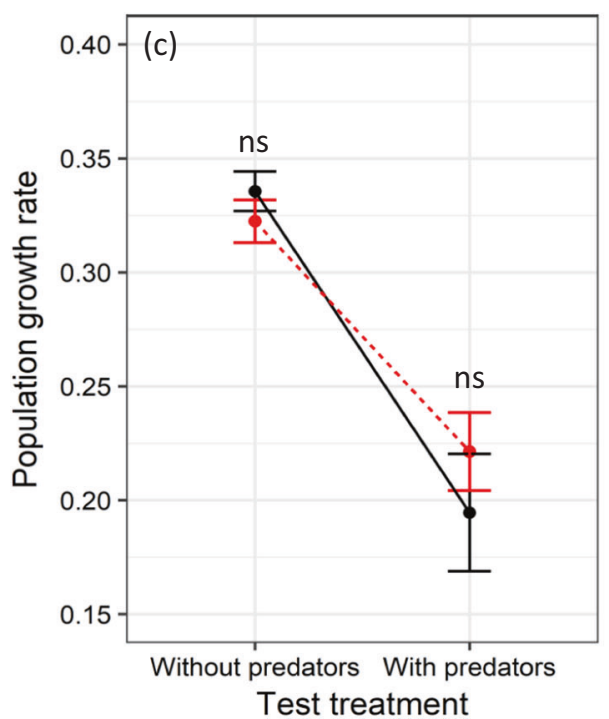

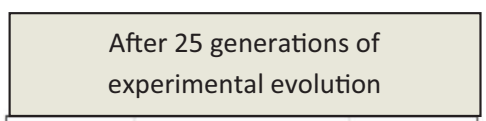

(b)
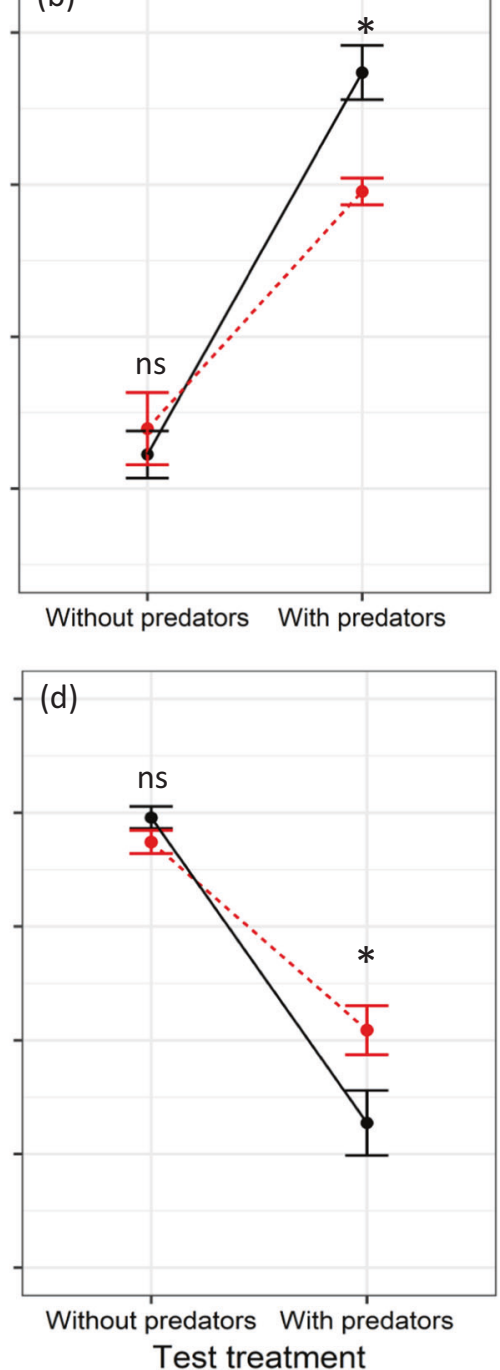

evolution, other than DNA sequence variation (Donelson et al. 2011; Donelson et al. 2018). However, the nongenetic evolution of induced phenotypes remains largely unexplored, which limits our understanding of the role of phenotypic plasticity in adaptive evolution. By using experimental evolution without standing genetic variation, we investigated changes in the transgenerational plastic response of aphids to their ladybird predators. We monitored the frequency of winged aphids over multiple generations submitted to a persistent predation pressure. We also removed the predation pressure at different stages of the experiment to test for changes in the persistence of the environmentally induced phenotype. Finally, at generations 16 or 25 of the experimental evolution, we conducted side experiments to compare the transgenerational plastic response to predators of naive aphids never exposed to predators and experienced aphids continuously exposed to predators. Our findings, that could only be revealed by experimental evolution over 27 generations, indicate that plasticity operates both in the short- and the long-term; and it is important to decompose processes acting at various timescales to determine the long-term phenotypic changes under a multigenerational exposure to environmental stimuli.

\section{Winged aphid frequency under persistent predation pressure}

In accordance with previous short-term studies (Dixon and Agarwala 1999; Weisser et al. 1999; Sentis et al. 2017b), we found a strong sudden increase (threefold) in the frequency of winged aphids after predators were introduced in 
the population. This frequency then dropped at the next generation before stabilizing at a higher level (twofold) than control lines for the 25 remaining generations (Fig. 1). The drop in winged frequency very likely resulted from the fact that winged females tend to produce very few winged offspring relative to unwinged ones (Sutherland 1970; MacKay 1977). At the second generation of experimental evolution, aphid lines exposed to predators were constituted of a majority $(\sim 80 \%)$ of winged females that produced fewer winged offspring resulting in a decrease in winged frequency. These results are consistent with theoretical models predicting that a negative maternal effect (winged aphids produce few winged aphids) initially reverses the response to selection (Kirkpatrick and Lande 1989). Although it remains to be investigated in more details, the stabilization of winged frequency to $\sim 45 \%$ probably reflects a balance between unwinged females producing many winged offspring and winged females producing fewer winged offspring.

\section{Winged aphid frequency after predator removal}

After removing predators in branch lines that were formerly separated from the main experiment, we observed complex but repeatable changes in phenotypic values. The frequency of winged aphids remained high for one generation before dropping abruptly below control levels, and then converging with the winged aphid frequencies of the control lines (Fig. 1). A high frequency of winged phenotypes persisted for only one generation after predator removal. This is likely reflecting the pre-natal determination of the winged phenotype reported for the pea aphid (Sutherland 1969), which might induce a transgenerational lag between the removal of predators and the decrease in the frequency of winged aphids. The short persistence of the winged phenotype is thus linked to the transgenerational induction of winged offspring and suggests that the induced winged phenotype is not inherited across generations. Our results thus contrast with other studies in Daphnia magna (Walsh et al. 2015) or Caenorhabditis elegans (Vastenhouw et al. 2006; Remy 2010; Devanapally et al. 2015; Klosin et al. 2017) where environmentally induced phenotypes persisted for many generations after the stimulus was removed. This persistence reached up to 14 generations in $C$. elegans (Klosin et al. 2017).

The following phase in the nonlinear change involved a drastic reduction of the frequency of winged aphids below the level of the control lines that were not exposed to predators. A third phase involved the return of phenotypic values to the level of control lines in a few generations. This nonlinear pattern was qualitatively similar at the three stages of the experimental evolution where branch lines were initiated, which supports its reproducibility. Previous studies showed that winged aphids tend to produce less winged aphids in response to crowding during the following two generations than wingless aphids (Sutherland 1970; MacKay 1977). This refractory phase may explain why, in our experiment, winged aphid frequencies remained below the control level for at least two generations.

\section{Reconstruction of the phenotypic response to predators}

The fact that the frequency of winged phenotypes remained high for only one generation after predator removal implies that the maintenance of a high frequency of winged aphids that we observed under permanent predation pressure directly resulted from the exposure to predators at every generation. In other words, the high frequency of winged aphid was not caused by stable heritable transmission but was reconstructed at every generation. To our knowledge, our study is the first to demonstrate that constant environmental stimuli lead to a rather stable reconstruction of the induced phenotype up to 25 generations (Fig. 1). However, although not intrinsically inherited, such a long-term reconstruction of the induced phenotype has the potential to shape evolutionary responses as it influences phenotypic diversity on which selection can act (Ghalambor et al. 2007; Ghalambor et al. 2015; Kuijper and Hoyle 2015). Additionally, once established through phenotypic plasticity, long-term environmentally induced variation might later become 'genetically assimilated' (Baldwin 1896; Waddington 1942, 1959; Lande 2009; Kronholm and Collins 2016).

\section{Change in plasticity after 16 and $\mathbf{2 5}$ generations of experimental evolution}

When comparing the plastic response to predators of naive aphids never exposed to predators before and experienced aphids continuously exposed to predators during the experimental evolution, we found that, after 16 generations of experimental evolution, permanent exposure to predators did not alter the aphid transgenerational plastic reaction norm. The naive and the experienced aphids did not show significant differences in their production of winged offspring in response to predators. Moreover, the population growth rates of naive and experienced aphids did not differ both in presence and absence of predators, indicating that, after 16 generations of experimental evolution, permanent exposure to predators was not sufficient to generate longlasting effects on this component of fitness of aphid populations. Contrastingly, after 25 generations of experimental evolution, experienced aphids produced $20 \%$ less winged offspring than naive aphids in the presence of predators, indicating a weaker transgenerational plastic response (i.e., 
flatter reaction norm) after a long period of selection against the production of winged offspring in our experimental system. As these additional experiments were conducted three generations after the removal of predators from the experimental evolution lines and when the population growth rate of predator removed lines did not significantly differ from the level of control lines, the observed decrease in plasticity was probably not explained by maternal and grand-maternal effects or by density-dependent population dynamical effects linked to differential population growth rate. Additionally, experienced aphids had a 19\% higher population growth rate than naïve aphids in the presence of predators. This result is consistent with the negative correlation between the population growth rate and winged aphid frequency. Our results therefore revealed an increase in the experimental population fitness in the presence of predators in association with the decrease in the plasticity of the winged phenotype frequency. This result is also consistent with the fact that winged individuals were selected against in our experimental setting (see Introduction), thus making the decrease in plasticity adaptive in our non-spatially structured experimental system. Our results therefore showcases an example of experimental evolution of plasticity in the absence of initial genetic variation in aphids and reveals that this non-genetic case of experimental phenotypic evolution had an adaptive component in this experimental context.

\section{Emergence of a long-term accumulation effect influencing winged frequency}

Through 27 generations of experimental evolution, we found three other pieces of evidence for modifications becoming more apparent with the growing number of generations of experimental evolution in the aphid lines exposed to persistent predation. First, there was a change in the persistence of the winged aphid frequency after predator removal: persistence declined gradually in strength with the number of generations of experimental evolution. Second, we also observed that, in the branch lines in which predators had been removed, the overall number of generations needed for winged aphid frequency to come back to control levels (i.e., the duration of the refractory phase) increased with the number of generations exposed to predators. This supports the hypothesis that the global nonlinear trajectory followed by the phenotypic value after predator removal was in itself affected by experimental evolution, because this refractory phase cumulatively became more and more long-lasting with the number of generations (Figs. 1 and 2). These findings are consistent with other studies showing that increasing the number of generations of exposure to environmental stimuli often tends to reinforce the strength and duration of non-genetic effects (Dombrovsky et al.
2009; Klosin et al. 2017). Another hypothesis that would explain the lengthening of the refractory phase with generation time in the absence of genetic variation builds on changes in aphid population growth rate. If the aphid replacement rate was reduced over time through experimental evolution, this could reduce the fraction of individuals that are beyond the refractory period, which would in turn delay the return to control levels. However, the support for this hypothesis is not large as (1) the population replacement rate was very high in our experiment (the probability of transferring an aphid twice was only 0.042) and (2) the length of the refractory phase differed between the second and third branch lines, while they were initiated at times when their population growth rates were similar in the control and predation lines. The third modification observed over the course of the experiment was the steeper negative correlation between winged frequency and population growth rate (Fig. 4b). This finding supports the hypothesis that constant predation over multiple generations changed the relationship between wing production and fitness. Collectively, these three findings suggest that a multigenerational exposure to predators gradually modified the non-genetic correlations between phenotypic traits in a way that suggests increased adaptation to the persistent predation pressure.

\section{Loss of plasticity and canalisation of the wingless phenotype}

Although, the frequency of winged aphid remained relatively constant across generations in the main long-term experiment, lines that were separated from predation revealed a modification of the plastic response between the 3rd and 25th generation and particularly so between the 16th and 25th generation of the experimental evolution. The factors explaining why winged aphid frequency remained relatively constant in the main long-term experiment whereas plasticity declined in the separated lines remain to be explored in more details. The apparent stability in winged frequency in the main experiment despite the persistent selection against winged individuals could be linked to environmental or demographic noise in the large experimental cages that have limited our ability to detect changes in winged frequency across generations. Another possibility is that grand-maternal and grand-grand-maternal effects linked to multigenerational predator exposure have contributed to the stability winged frequency in the main experimental lines. These grand-maternal and grand-grandmaternal effects were controlled for in lines separated from the main experiment as we removed predators for three generations before assessing the aphid plastic response to predators. Whatever the exact mechanisms explaining this difference, the lengthening of the refractory phase, the 
decrease of the winged phenotype persistence, and the weaker plastic response to predators are all consistent with a selection against winged individuals in lines constantly exposed to predators. Under such selection pressure, we can expect persistent exposure to predation to reduce plasticity, ultimately canalising the trait towards the wingless phenotype. This wingless phenotype might then become constitutive either through strong genetic or non-genetic encoding leading to parent-offspring similarity. Our results indicate that the decrease of plasticity started to occur after at least 13 generations of constant exposure to predators, which suggest that the canalisation of the wingless phenotype is likely to take many generations unless a qualitative shift in the dynamic of the system occurs (e.g., emergence of genetic mutations speeding the process of canalisation).

\section{Limits of the study and potential molecular mechanisms underlying the observed phenotypic changes}

As often with laboratory studies, our small-scale, mid-term experiment was conducted in an artificial environment that has little in common with natural conditions, which limits the ecological relevance of our findings and their generalisation. This does not however undermine their value as a fundamental test for the experimental evolution of plasticity in the absence of initial standing genetic variation. A first limit was that we used only one aphid clone because of logistical constraints. As the magnitude of the plastic response to predators as well as the duration of the refractory phase can vary from clone to clone (Sutherland 1970; MacKay 1977), it is thus impossible to assess whether our findings would have been similar for other aphid clones. However, this limitation does not forbid us to conclude that evolution very likely can occur in the absence of initial standing genetic variation, which was the main question justifying this experiment. Second, in the absence of a temporal replicate of the experiment, we cannot rule out the potential influence of an uncontrolled change in the experimental conditions across time (e.g., changes in atmospheric pressure or water quality across the experiment) on the experimental results. The influence of external factors was nevertheless limited because the experiment was conducted under tightly controlled laboratory conditions. Third, and more importantly, we did not investigate the molecular mechanism(s) underlying the observed phenotypic changes. In the peach-potato aphid, Myzus persicae, some clones resistant to insecticides were shown to revert to sensitivity rapidly (i.e., 3-4 generations) after removal of insecticidal pressure. This loss of resistance was associated with the loss of methylation in amplified esterase genes responsible for hydrolysis and sequestration of insecticides (Field et al. 1989; Hick et al. 1996). On the other hand,
Gorokhova et al. (2002) reported that artificial selection on weight-specific fecundity in clonal lineages of Daphnia pulex derived from a single female can lead, in less than five generations, to an increased predominance of long variants of the intergenic spacer of rDNA, indicating a genetic mechanism for rapid adaptive change in major life history traits. Altogether, these studies indicate that both genetic and non-genetic mechanisms can operate on relatively short timescales (i.e., a few generations). It would thus be interesting to compare the genetic and epigenetic profiles of aphids before, during, and after the refractory phase at different time points of the experimental evolution to better understand which molecular mechanisms underpin the observed phenotypic changes. Although, the occurrence of DNA mutations during the course of our experimental evolution cannot be excluded, they are unlikely to explain our findings. This is because the random occurrence in each of the nine independent experimental lines of DNA mutations with similar effects that then changed similarly in frequency seems very unlikely. Additionally, the fact that the winged frequencies in the branch lines always fell back to the control level after a few generations is far from matching the high stability of DNA sequence information and the very low probability of reverse DNA mutations. We can thus tentatively attribute the decline in plasticity observed in lines that were exposed to predators for many generations to the action of some non-genetically transmitted information (i.e. information not encoded in the DNA sequence). The hypothesis that observed phenotypic changes were caused by reversible epigenetic changes is thereby more likely but in order to be confirmed, this hypothesis would require to be backed up by molecular analyses.

\section{Conclusion}

We found that the persistent exposure to environmental stimuli drove a rather stable reconstruction of the environmentally induced phenotype frequency over many generations (i.e. $>25$ ). This stability implies that in this system, the plastic response to predators might be consistent over time, which, in turn, yields to a high frequency of phenotypic (non-genetic) variants upon which selection can act. Nonetheless, our results on branch lines where predators were removed at generations 3,13 , and 22 , as well as the comparison of the fitness and plasticity of experimental evolution lines at generations 16 and 25 reveal gradual changes with the number of generations of predation exposure for: (1) the persistence of the winged phenotype after predator removal, (2) the duration of the refractory phase (i.e., the number of generations needed for winged aphid frequency to come back to control levels after 
predator removal), (3) the plastic response to predator exposure and (4) the fitness of aphid populations. These results are consistent with a selection against wing production in our non-spatially structured experimental system. Given the pace of the observed trends of change, we suspect that the observed gradual changes involved the action of reversible epigenetic mechanisms. More generally, our findings imply that integrating the various dimensions of transgenerational plasticity (e.g. changes in refractory phases, in reaction norms and in non-genetic correlations) has the potential to reveal evolutionary changes where they would not have been detected at first (e.g. stable phenotypic reconstruction of the trait value in a specific environment). Our results suggest that experimental evolution in the absence of initial genetic variation constitute a major research approach to explore the evolutionary role of nongenetic inheritance.

\section{Data availability}

Data available from the Dryad Digital Repository: https:// doi.org/10.5061/dryad.34q3p09.

Acknowledgements We thank Foteini Spagopoulou, Martin Lind, and four anonymous reviewers for the detailed comments that substantially improved the manuscript. This work was supported by the ANR funded French Laboratory of Excellence projects 'LABEX TULIP' and 'LABEX CEBA' (ANR-10-LABX-41, ANR-10-LABX-25-01), the Réseau Thématique Pluridisciplinaire "Epigenetics in Ecology and Evolution" (RTP3E), and ANR funded Toulouse Initiative of Excellence "IDEX UNITI" (ANR11-IDEX-0002-02). A.S. was also founded by the People Program (Marie Curie Actions) of the European Union's Seventh Framework Program (FP7/2007-2013) under REA grant agreement $n{ }^{\circ}$ PCOFUND-GA-2013-609102, through the PRESTIGE program coordinated by Campus France.

\section{Compliance with ethical standards}

Conflict of interest The authors declare that they have no conflict of interest.

\section{References}

Agrawal AA (2001) Phenotypic plasticity in the interactions and evolution of species. Science 294:321-326

Andrade MC, Roitberg BD (1995) Rapid response to intraclonal selection in the pea aphid (Acyrthosiphon pisum). Evolut Ecol 9:397-410

Bakdash JZ, Marusich LR (2017) Repeated measures correlation. Front Psychol 8:456

Baldwin JM (1896) A new factor in evolution. Am Nat 30:441-451

Bates D, Mächler M, Bolker B, Walker S (2015) Fitting linear mixedeffects models using lme4. J Stat Softw 67:1-48

Bonduriansky R (2012) Rethinking heredity, again. Trends Ecol Evol 27:330-336

Bossdorf O, Richards CL, Pigliucci M (2008) Epigenetics for ecologists. Ecol Lett 11:106-115

Boyko A, Blevins T, Yao Y, Golubov A, Bilichak A, Ilnytskyy Y et al. (2010) Transgenerational adaptation of Arabidopsis to stress requires DNA methylation and the function of Dicer-like proteins. PLoS ONE 5:e9514

Brisson JA (2010) Aphid wing dimorphisms: linking environmental and genetic control of trait variation. Philos Trans R Soc B: Biol Sci 365:605-616

Burton T, Metcalfe NB (2014) Can environmental conditions experienced in early life influence future generations? Proc R Soc Lond B: Biol Sci 281:20140311

Chevin L.-M, Lande R, Mace GM (2010) Adaptation, plasticity, and extinction in a changing environment: towards a predictive theory. PLoS Biol 8:e1000357

Cortijo S, Wardenaar R, Colomé-Tatché M, Gilly A, Etcheverry M, Labadie K et al. (2014) Mapping the epigenetic basis of complex traits. Science 343:1145-1148

Danchin É (2013) Avatars of information: towards an inclusive evolutionary synthesis. Trends Ecol Evol 28:351-358

Danchin É, Charmantier A, Champagne FA, Mesoudi A, Pujol B, Blanchet S (2011) Beyond DNA: integrating inclusive inheritance into an extended theory of evolution Nat Rev Genet 12:475-486

Danchin É, Pocheville A, Rey O, Pujol B, Blanchet S (2018) Epigenetically-facilitated mutational assimilation: epigenetics as a hub within the inclusive evolutionary synthesis. Biol Rev, In press.

Danchin É, Wagner RH (2010) Inclusive heritability: combining genetic and non-genetic information to study animal behavior and culture Oikos 119:210-218

Day T, Bonduriansky R (2011) A unified approach to the evolutionary consequences of genetic and nongenetic inheritance. Am Nat 178:E18-E36

de Vos M, Cheng WY, Summers HE, Raguso RA, Jander G (2010) Alarm pheromone habituation in Myzus persicae has fitness consequences and causes extensive gene expression changes. Proc Natl Acad Sci USA 107:14673-14678

Devanapally S, Ravikumar S, Jose AM (2015) Double-stranded RNA made in C. elegans neurons can enter the germline and cause transgenerational gene silencing. Proc Natl Acad Sci USA 112:2133-2138

Dias BG, Maddox SA, Klengel T, Ressler KJ (2015) Epigenetic mechanisms underlying learning and the inheritance of learned behaviors. Trends Neurosci 38:96-107

Dias BG, Ressler KJ (2014) Parental olfactory experience influences behavior and neural structure in subsequent generations. Nat Neurosci 17:89-96

Dixon AFG, Agarwala BK (1999) Ladybird-induced life-history changes in aphids. Philos Trans $\mathrm{R}$ Soc $\mathrm{B}$ : Biol Sci 266:1549-1553

Dixon AFG, Horth S, Kindlmann P (1993) Migration in insects: cost and strategies. J Animal Ecol 62:182-190

Dombrovsky A, Arthaud L, Ledger TN, Tares S, Robichon A (2009) Profiling the repertoire of phenotypes influenced by environmental cues that occur during asexual reproduction. Genome Res 19:2052-2063

Donelson J, Munday P, McCormick M, Pitcher C (2011) Rapid transgenerational acclimation of a tropical reef fish to climate change. Nat Clim Change 2:30-32

Donelson JM, Salinas S, Munday PL, Shama LNS (2018) Transgenerational plasticity and climate change experiments: where do we go from here? Glob Change Biol 4:13-34

Feil R, Fraga MF (2012) Epigenetics and the environment: emerging patterns and implications. Nat Rev Genet 13:97-109

Field LM, Devonshire AL, Ffrench-Constant RH, Forde BG (1989) Changes in DNA methylation are associated with loss of insecticide resistance in the peach-potato aphid Myzus persicae (Sulz.). FEBS Lett 243:323-327 
Gardner JL, Peters A, Kearney MR, Joseph L, Heinsohn R (2011) Declining body size: a third universal response to warming? Trends Ecol \& Evol 26:285-291

Ghalambor CK, Hoke KL, Ruell EW, Fischer EK, Reznick DN, Hughes KA (2015) Non-adaptive plasticity potentiates rapid adaptive evolution of gene expression in nature. Nature 525:372-375

Ghalambor CK, McKay JK, Carroll SP, Reznick DN (2007) Adaptive versus non-adaptive phenotypic plasticity and the potential for contemporary adaptation in new environments. Funct Ecol 21:394-407

Gorokhova E, Dowling TE, Weider LJ, Crease TJ, Elser JJ (2002) Functional and ecological significance of rDNA intergenic spacer variation in a clonal organism under divergent selection for production rate. Proc R Soc Lond B: Biol Sci 269:2373-2379

Gotelli N, Ellison A (2004) A primer of ecological statistics.. Sinauer Associates, Sunderland, Massachusetts, USA

Herman J, Sultan S (2011) Adaptive transgenerational plasticity in plants: case studies, mechanisms, and implications for natural populations. Front Plant Sci 2:1-10

Herman JJ, Spencer HG, Donohue K, Sultan SE (2014) How stable "should" epigenetic modifications be? insights from adaptive plasticity and bet hedging. Evolution 68:632-643

Hick CA, Field LM, Devonshire AL (1996) Changes in the methylation of amplified esterase DNA during loss and reselection of insecticide resistance in peach-potato aphids, Myzus persicae. Insect Biochem Mol Biol 26:41-47

Holeski LM, Jander G, Agrawal AA (2012) Transgenerational defense induction and epigenetic inheritance in plants. Trends Ecol Evol 27:618-626

Huey RB, Kearney MR, Krockenberger A, Holtum JA, Jess M, Williams SE (2012) Predicting organismal vulnerability to climate warming: roles of behaviour, physiology and adaptation. Philos Trans R Soc B: Biol Sci 367:1665-1679

Huneman P, Walsh D (2017) Challenging the Modern Synthesis: Adaptation, Development, and Inheritance.. Oxford University Press, New York, USA

Johannes F, Colot V, Jansen RC (2008) Epigenome dynamics: a quantitative genetics perspective. Nat Rev Genet 9:883-890

Kawecki TJ, Lenski RE, Ebert D, Hollis B, Olivieri I, Whitlock MC (2012) Experimental evolution. Trends Ecol Evol 27:547-560

Kirkpatrick M, Lande R (1989) The evolution of maternal characters. Evolution 43:485-503

Klosin A, Casas E, Hidalgo-Carcedo C, Vavouri T, Lehner B (2017) Transgenerational transmission of environmental information in C. elegans. Science 356:320-323

Kronholm I, Collins S (2016) Epigenetic mutations can both help and hinder adaptive evolution. Mol Ecol 25:1856-1868

Kuijper B, Hoyle RB (2015) When to rely on maternal effects and when on phenotypic plasticity? Evolution 69:950-968

Lande R (2009) Adaptation to an extraordinary environment by evolution of phenotypic plasticity and genetic assimilation. J Evolut Biol 22:1435-1446

Luquet E, Tariel J (2016) Offspring reaction norms shaped by parental environment: interaction between within- and trans-generational plasticity of inducible defenses. BMC Evolut Biol 16:209

MacKay PA (1977) Alata-production by an aphid: the "interval timer" concept and maternal age effects. J Insect Physiol 23:889-893

Miner BG, Sultan SE, Morgan SG, Padilla DK, Relyea RA (2005) Ecological consequences of phenotypic plasticity. Trends Ecol Evol 20:685-692

Munday PL, Warner RR, Monro K, Pandolfi JM, Marshall DJ (2013) Predicting evolutionary responses to climate change in the sea. Ecol Lett 16:1488-1500

Pasquier C, Clément M, Dombrovsky A, Penaud S, Da Rocha M, Rancurel C et al. (2014) Environmentally selected aphid variants in clonality context display differential patterns of methylation in the genome. PLoS ONE 9:e115022

Pocheville A, Danchin É (2017) Genetic assimilation and the paradox of blind variation. In: Humeman P, Walsh D (eds) Challenging the Modern Synthesis. Oxford University press, Oxford, pp $111-136$

Pujol B, Blanchet S, Charmantier A, Danchin E, Facon B, Marrot P et al. (2018) The missing response to selection in the wild. Trends Ecol Evol 33:337-346

R Development Core Team (2017). $R$ : a language and environment for statistical computing, R Foundation for Statistical Computing, Vienna, Austria.

Rechavi O, Minevich G, Hobert O (2011) Transgenerational inheritance of an acquired small RNA-based antiviral response in $C$. elegans. Cell 147:1248-1256

Remy J.-J (2010) Stable inheritance of an acquired behavior in Caenorhabditis elegans. Curr Biol 20:R877-R878

Schott D, Yanai I, Hunter CP (2014) Natural RNA interference directs a heritable response to the environment. Sci Rep 4:7387

Sentis A, Binzer A, Boukal DS (2017a) Temperature-size responses alter food chain persistence across environmental gradients. Ecol Lett 20:852-862

Sentis A, Hemptinne J.-L, Brodeur J (2017b) Non-additive effects of simulated heat waves and predators on prey phenotype and transgenerational phenotypic plasticity. Glob Change Biol 23:4598-4608

Sentis A, Morisson J, Boukal DS (2015) Thermal acclimation modulates the impacts of temperature and enrichment on trophic interaction strengths and population dynamics. Glob Change Biol 21:3290-3298

Srinivasan DG, Brisson JA (2012) Aphids: a model for polyphenism and epigenetics. Genet Res Int 2012:1-12

Sutherland O (1970) An intrinsic factor influencing alate production by two strains of the pea aphid. Acyrthosiphon pisum J Insect Physiol 16:1349-1354

Sutherland ORW (1969) The role of crowding in the production of winged forms by two strains of the pea aphid, Acyrthosiphon pisum. J Insect Physiol 15:1385-1410

Tollrian R, Harvell CD (1999) The ecology and evolution of inducible defenses. Princeton University Press, Princeton, NJ, USA

Turner BM (2009) Epigenetic responses to environmental change and their evolutionary implications. Philos Trans R Soc Lond B: Biol Sci 364:3403-3418

Vastenhouw NL, Brunschwig K, Okihara KL, Müller F, Tijsterman M, Plasterk RH (2006) Gene expression: long-term gene silencing by RNAi. Nature 442:882-882

Vellichirammal NN, Gupta P, Hall TA, Brisson JA (2017) Ecdysone signaling underlies the pea aphid transgenerational wing polyphenism. Proc Natl Acad Sci USA 114:1419-1423

Vellichirammal NN, Madayiputhiya N, Brisson JA (2016) The genomewide transcriptional response underlying the pea aphid wing polyphenism. Mol Ecol 25:4146-4160

Waddington CH (1942) Canalization of development and the inheritance of acquired characters. Nature 150:563-565

Waddington $\mathrm{CH}$ (1959) Canalization of development and genetic assimilation of acquired characters. Nature 183:1654-1655

Walsh MR, Cooley F, Biles K, Munch SB (2015) Predator-induced phenotypic plasticity within- and across-generations: a challenge for theory? Proc R Soc B: Biol Sci 282:20142205

Walsh TK, Brisson JA, Robertson HM, Gordon K, Jaubert-Possamai S, Tagu D et al. (2010) A functional DNA methylation system in the pea aphid, Acyrthosiphon pisum. Insect Mol Biol 19:215-228

Wang Y, Liu H, Sun Z (2017) Lamarck rises from his grave: parental environment-induced epigenetic inheritance in model organisms and humans. Biol Rev 92:2084-2111 
Weisser W, Braendle C, Minoretti N (1999) Predator-induced morphological shift in the pea aphid. Proc R Soc B: Biol Sci 266:1175-1181

West-Eberhard MJ (2003) Developmental plasticity and evolution.. Oxford University Press, New York

Whitman DW, Agrawal AA (2009) What is phenotypic plasticity and why is it important? In: Whitman DW, Ananthakrishnan TN eds. Phenotypic plasticity of insects. Science Publishers, Enfield, USA, p 1-63
Whittle C, Otto S, Johnston MO, Krochko J (2009) Adaptive epigenetic memory of ancestral temperature regime in Arabidopsis thaliana. Botany 87:650-657

Zera AJ (2003) The endocrine regulation of wing polymorphism in insects: state of the art, recent surprises, and future directions. Integr Comp Biol 43:607-616 